\title{
Can a strongly interacting Higgs boson rescue SU(5)?
}

\author{
Matthew H. Austern \\ Lawrence Berkeley Laboratory, University of California, Berkeley, California, 94720 \\ and \\ Physics Department, University of California at Berkeley, Berkeley, California, 94720
}

(Received January 3, 2018)

\begin{abstract}
Renormalization group analyses show that the three running gauge coupling constants of the Standard Model do not become equal at any energy scale. These analyses have not included any effects of the Higgs boson's self-interaction. In this paper, I examine whether these effects can modify this conclusion.
\end{abstract}

PACS numbers: 12.10.Dm, 11.10.Jj, 14.80.Gt

Typeset Using REVTEX 
Although the Standard Model, a gauge theory based on the gauge group $\mathrm{SU}(3) \times \mathrm{SU}(2) \times$ $\mathrm{U}(1)$, has experimentally proved to be very successful, one unattractive feature is that this gauge group is not simple, i.e., that the theory has three independent coupling constants, which are conventionally denoted $g_{3}, g_{2}$, and $g_{1}$. One possible solution is that this gauge group is merely the low-energy manifestation of a simple Lie group, which is spontaneously broken to $\mathrm{SU}(3) \times \mathrm{SU}(2) \times \mathrm{U}(1)$ at some high energy scale. A generic feature of these "grand unified theories" (GUTs) is that the three coupling constants evaluated at this scale are numerically equal.

At the one-loop level, it is straightforward to calculate the $\beta$-functions of the three Standard Model gauge coupling constants; the well-known result [1] is

$$
\mu \frac{d g_{i}}{d \mu}=-\frac{b_{i}}{16 \pi^{2}} g_{i}^{3}
$$

where, for $N$ generations of fermions and $n_{h}$ doublets of Higgs scalars,

$$
\begin{aligned}
& b_{1}=-\frac{4}{3} N-\frac{1}{10} n_{h} \\
& b_{2}=\frac{22}{3}-\frac{4}{3} N-\frac{1}{6} n_{h} \\
& b_{3}=11-\frac{4}{3} N .
\end{aligned}
$$

Integrating these equations,

$$
\alpha_{i}^{-1}(\mu)=\frac{b_{i}}{2 \pi} \ln \left(\frac{\mu}{\mu_{0}}\right)+\alpha_{i}^{-1}\left(\mu_{0}\right),
$$

where $\mu_{0}$ is some scale at which the coupling constants are known.

The initial renormalization point, $\mu_{0}$, is arbitrary; it is convenient to choose it to be $M_{Z}$. The strong coupling constant, $\alpha_{3}\left(M_{Z}\right)$, can be extracted from a variety of experiments; the Particle Data Group [2] reports the average $\alpha_{3}\left(M_{Z}\right)=0.1134 \pm 0.0035$. The electroweak coupling constants, $\alpha_{1}\left(M_{Z}\right)$ and $\alpha_{2}\left(M_{Z}\right)$, are obtained by the LEP measurements of $\alpha_{E M}\left(M_{Z}\right)$ and $\sin ^{2} \theta_{\overline{\mathrm{MS}}}$, yielding [3]

$$
\begin{aligned}
& \alpha_{1}\left(M_{Z}\right)=0.016887 \pm 0.000040 \\
& \alpha_{2}\left(M_{Z}\right)=0.03322 \pm 0.00025
\end{aligned}
$$

Note that $\alpha_{1}$ is normalized in the way that is conventional for discussions of GUTs, not in the way that is conventional for discussions of the Standard Model. That is,

$$
\alpha_{1}\left(M_{Z}\right)=\frac{5}{3} \frac{\alpha_{E M}\left(M_{Z}\right)}{\cos ^{2} \theta_{\overline{\mathrm{MS}}}} .
$$

A different choice of normalization would require both a different $\beta$-function and different matching conditions at the GUT scale.

Using the central values of these coupling constants, and assuming three generations of fermions and a single Higgs doublet, Eq. (5) is plotted in Fig. 1. Note that the three 
couplings do not meet at a single point; unification is excluded [3] by more than 7 standard deviations.

A more careful analysis [4,5] would include two-loop effects and matching conditions, but these effects turn out to be small corrections [6], and the essential result is unchanged. A GUT is thus only possible if an intermediate unification scale exists, or if additional physics, such as supersymmetry, enters into the $\beta$-function, Eq. (11). The simplest GUT, $\mathrm{SU}(5)$, possesses no intermediate scale, so these results are usually assumed to exclude nonsupersymmetric $\mathrm{SU}(5)$ with the minimal scalar sector.

In the Standard Model, it is well known that if the Higgs boson is very massive, its self-interactions become strong. None of the renormalization-group analyses of the Standard Model include the Higgs self-interaction, so it is interesting to consider whether this might in fact be the additional physics that is necessary to achieve unification. This selfinteraction cannot affect the one-loop calculation of the gauge coupling $\beta$-functions, but, if it is sufficiently strong, it might be significant even as a higher-order effect.

Using the axial gauge, the renormalization of a gauge coupling constant is related to the gauge boson's wave-function renormalization by the Ward identity

$$
Z_{g}=\frac{1}{\sqrt{Z_{A}}}
$$

so it suffices to consider vacuum polarization diagrams. The number of diagrams is further reduced by regarding $\mathrm{SU}(2) \times \mathrm{U}(1)$ as a theory with unbroken gauge symmetry. At an energy scale $\mu$, any effect that depends on symmetry breaking will be suppressed by some power of $v / \mu$, where $v \approx 246 \mathrm{GeV}$ is the vacuum expectation value of the Higgs field. As we are dealing with scales up to $10^{16} \mathrm{GeV}$, this suppression factor renders such effects completely negligible.

In the Standard Model, the Higgs scalars do not couple to gluons. The lowest-order diagrams that might affect the $\mathrm{SU}(2)$ or $\mathrm{U}(1) \beta$-functions are given in Fig. 2. In fact, however, neither of these diagrams contributes to the wave-function renormalization: Such contributions are only from divergent terms that are momentum-dependent and that have the Lorentz structure $g^{\mu \nu}$, and it is clear that neither of these diagrams has such a form. The lowest-order nonvanishing contributions are three-loop diagrams, which are shown in Fig. 3. These diagrams have been evaluated by Curtright [7] and Jones [8].

In Curtright's notation, the scalar self-interaction is given by

$$
\mathcal{L}_{q}=-\frac{\lambda}{4 !} F_{i j k l} \phi_{i} \phi_{j} \phi_{k} \phi_{l}
$$

where $\phi$ is a multiplet of real scalar fields. Its covariant derivative is given by

$$
\left(\mathcal{D}_{\mu} \phi\right)_{i}=\partial_{\mu} \phi_{i}+i g A_{\mu}^{a}\left(T^{a}\right)_{i j} \phi_{j}
$$

where $T^{a}$ is a representation matrix of the gauge group. The contribution of $\mathcal{L}_{q}$ to the $\beta$-function, then, is

$$
\beta_{q}=\frac{1}{4 !}\left(\frac{1}{16 \pi^{2}}\right)^{3} g^{3} \lambda^{2} T F
$$

where 


$$
T \equiv \operatorname{Tr}\left(T^{a}\right)^{2}
$$

and $F$ is defined by

$$
F_{i k l m} F_{j k l m} \equiv F \delta_{i j}
$$

In the minimal Standard Model with one Higgs doublet, the quartic scalar self-interaction is given by

$$
\mathcal{L}_{q}=\lambda\left(|\Phi|^{2}\right)^{2}
$$

where the relation between $\lambda$ and the mass of the physical Higgs boson is

$$
m_{H}=v \sqrt{2 \lambda(v)}
$$

For this model, $F=72, T_{\mathrm{SU}(2)}=1$, and $T_{\mathrm{U}(1)}=25 / 9$.

The contributions to the $\mathrm{SU}(2)$ and $\mathrm{U}(1) \beta$-functions, then, are

$$
\beta_{q}=\frac{24 \pi}{\left(16 \pi^{2}\right)^{3}} \alpha_{i}^{2} \lambda^{2} T_{i}
$$

This contribution to the running coupling may be calculated by rewriting the renormalization group equation as an integral equation, and solving it by iteration. The one-loop result has been given in Eq. 5, and the correction from Eq. 17 is given by

$$
\begin{aligned}
\delta \alpha_{i}(\mu) & =\frac{24 \pi T_{i}}{\left(16 \pi^{2}\right)^{3}} \int_{0}^{\mu / \mu_{0}} d t \alpha_{i}^{2}\left(\mu_{0} e^{t}\right) \lambda^{2}\left(\mu_{0} e^{t}\right) \\
& \approx \frac{24 \pi T_{i}}{\left(16 \pi^{2}\right)^{3}} \int_{0}^{\mu / \mu_{0}} d t \frac{\lambda^{2}\left(\mu_{0} e^{t}\right)}{\left(\frac{b_{i}}{2 \pi} t+\alpha_{i}^{-1}\left(\mu_{0}\right)\right)^{2}}
\end{aligned}
$$

If the running of $\lambda$ can be neglected, i.e., if $\lambda(\mu) \approx \lambda\left(\mu_{0}\right)$, this integral can easily be evaluated, yielding

$$
\delta \alpha_{i}(\mu)=\frac{48 \pi^{2} \lambda^{2} T_{i}}{\left(16 \pi^{2}\right)^{3}} \frac{\alpha_{i}^{2}\left(\mu_{0}\right) \ln \left(\frac{\mu}{\mu_{0}}\right)}{2 \pi+b_{i} \alpha_{i}\left(\mu_{0}\right) \ln \left(\frac{\mu}{\mu_{0}}\right)} .
$$

If $\lambda$ is taken to be the largest value that could possibly be reasonable, i.e., $\lambda=4 \pi$, which corresponds to $m_{H} \approx 1.2 \mathrm{TeV}$, then

$$
\begin{aligned}
& \delta \alpha_{1}\left(10^{15} \mathrm{GeV}\right)=1.1 \times 10^{-4} \\
& \delta \alpha_{2}\left(10^{15} \mathrm{GeV}\right)=6.7 \times 10^{-5}
\end{aligned}
$$

This shift is too small to have a significant effect on the failure of coupling constant unification.

In fact, even these numbers are unrealistically large, because the running of $\lambda$ cannot be neglected. In theories with self-interacting scalar fields, the running scalar coupling constant increases with the energy scale, eventually diverging at some finite scale. It appears, although 
it has not been proved, that if a scalar field theory is to be valid for arbitrary high scales, the coupling constant must vanish [9].

If "triviality" is a real phenomenon and not merely an artifact of computational schemes, then scalar field theories must be regarded as effective field theories, valid only up to some cutoff $\Lambda$. The larger $\Lambda$ is taken to be, the smaller are the permissable values of the coupling constant $\lambda$, and as $\Lambda$ is taken to $\infty, \lambda$ is driven to zero.

The $\beta$-function for $\lambda$ may be approximated by neglecting the contribution from gauge boson and fermion loops; this is reasonable, since the domain of interest is when $\lambda$ is large. The one-loop result is

$$
\mu \frac{d \lambda}{d \mu}=\frac{3}{2 \pi^{2}} \lambda^{2}
$$

and

$$
\lambda(\mu)=\frac{1}{\lambda^{-1}\left(\mu_{0}\right)-\frac{3}{2 \pi^{2}} \ln \left(\frac{\mu}{\mu_{0}}\right)} .
$$

Requiring that $\lambda$ be finite up to the cutoff $\Lambda$ yields the upper bound

$$
\lambda\left(\mu_{0}\right) \leq \frac{2 \pi^{2}}{3} \frac{1}{\ln \left(\frac{\Lambda}{\mu_{0}}\right)} .
$$

In particular, in the minimal $\mathrm{SU}(5)$ model, the cutoff $\Lambda$ must be at least the GUT scale. Taking $\Lambda=10^{16} \mathrm{GeV}$ then implies $\lambda(v) \leq 0.21$, and $m_{H} \leq 160 \mathrm{GeV}$. Experience with other techniques [9,6] suggests that this simple one-loop result is at least qualitatively correct.

Taking the running of $\lambda$ into account, Eq. 19 becomes

$$
\begin{aligned}
\delta \alpha_{i}(\mu)= & \frac{24 \pi T_{i}}{\left(16 \pi^{2}\right)^{3}} \int_{0}^{\mu / \mu_{0}} \frac{d t}{\left(\alpha_{i}^{-1}\left(\mu_{0}\right)+\frac{b_{i}}{2 \pi} t\right)^{2}\left(\lambda^{-1}\left(\mu_{0}\right)-\left(\frac{3}{2 \pi^{2}}\right) t\right)^{2}} \\
= & \frac{3 T_{i}}{(16 \pi)^{2}} \frac{\alpha_{i}^{2}\left(\mu_{0}\right) \lambda^{2}\left(\mu_{0}\right)}{3 \lambda\left(\mu_{0}\right)+\alpha_{i}\left(\mu_{0}\right) b_{i} \pi}\left[\frac{b_{i} \alpha_{i}^{2}\left(\mu_{0}\right) t}{2 \pi+b_{i} \alpha_{i}\left(\mu_{0}\right) t}+\frac{9}{\pi} \frac{\lambda^{2}\left(\mu_{0}\right) t}{2 \pi^{2}-3 \lambda\left(\mu_{0}\right) t}\right. \\
& \left.\quad+\frac{6 b_{i} \alpha_{i}\left(\mu_{0}\right) \lambda\left(\mu_{0}\right)}{3 \lambda\left(\mu_{0}\right)+\alpha_{i}\left(\mu_{0}\right) b_{i} \pi} \ln \left(\frac{2 \pi^{2}+\alpha_{i}\left(\mu_{0}\right) b_{i} \pi t}{2 \pi^{2}-3 \lambda\left(\mu_{0}\right) t}\right)\right],
\end{aligned}
$$

where $t=\ln \left(\mu / \mu_{0}\right)$. Taking $\lambda\left(M_{Z}\right)=0.2$ in accordance with the "triviality" limit, $\delta \alpha$ is plotted in Fig. 4. Note that $\delta \alpha$ is completely negligible until extremely close to the scale where $\lambda$ diverges. At this scale, however, this calculation no longer makes sense: By definition, this is the scale at which the physics of the Higgs sector can no longer adequately be described by a scalar field.

The contribution from the Higgs self-interaction is too small to affect the conclusion that the minimal SU(5) model is ruled out. This contribution can, however, potentially be as large as some of the two-loop terms, and should be included in any precision analyses. 


\section{ACKNOWLEDGMENTS}

I wish to thank Robert Cahn and Lawrence Hall for helpful discussions. This work was supported by the Director, Office of Energy Research, Office of High Energy and Nuclear

Physics, Division of High Energy Physics of the U.S. Department of Energy under Contract DE-AC03-76SF00098. 


\section{REFERENCES}

[1] D. J. Gross and F. Wilczek, Phys. Rev. Lett. 30, 1343 (1973); H. D. Politzer, Phys. Rev. Lett. 30, 1346 (1973);

[2] Particle Data Group, M. Aguilar-Benitez, et al, Phys. Rev. D45, Part 2 (1992).

[3] U. Amaldi, W. de Boer, and H. Fürstenau, Phys. Lett. B260, 447 (1991).

[4] L. Hall, Nucl. Phys. B178, 75 (1981).

[5] D. R. T. Jones, Phys. Rev. D25, 581 (1982).

[6] H. Arason, et al, "Renormalization group study of the Standard Model and its extensions: I. The Standard Model," UFIFT-HEP-91-33, 1992. (unpublished)

[7] T. Curtright, Phys. Rev. D21, 1543 (1980).

[8] D. R. T. Jones, Phys. Rev. D22, 3140 (1980).

[9] See, for example, D. J. E. Callaway, Phys. Rept. 167, 241 (1988). 


\section{FIGURES}

FIG. 1. One-loop calculation of the running of the $\mathrm{SU}(3), \mathrm{SU}(2)$, and $\mathrm{U}(1)$ coupling constants, assuming that the low-energy particle content is a single complex scalar doublet and three generations of quarks and leptons. The three lines represent the central values of the coupling constants, and the shaded regions represent the one- $\sigma$ errors. Note that although they approach a similar order of magnitude at high energies, the three constants never become equal.

FIG. 2. Two-loop diagrams involving the four-point scalar self-interaction term. The external gauge bosons are those of $\mathrm{SU}(2) \times \mathrm{U}(1)$. Neither diagram contributes to the vacuum polarization, nor, as a consequence, to the $\beta$-function.

FIG. 3. Three-loop diagrams involving the four-point scalar self-interaction. The external gauge bosons are those of $\mathrm{SU}(2) \times \mathrm{U}(1)$. These diagrams are the lowest-order contribution to the $\mathrm{SU}(2)$ and $\mathrm{U}(1)$ gauge coupling $\beta$-functions that involves that interaction.

FIG. 4. Contribution to the running of $\alpha_{2}$, the $\mathrm{SU}(2)$ coupling constant, from the Higgs self-interaction. The quartic coupling constant $\lambda$ has been taken to be 0.2 , which is the maximum possible value if the theory is to be valid up to $10^{16} \mathrm{GeV}$, and is evolved according to the one-loop $\beta$-function. Note that this contribution to $\alpha$ is negligible until the scale where $\lambda$ diverges. 